\title{
Asymptotic Nusselt and entropy generation numbers for non-Newtonian fluid flow
}

\author{
Shohel Mahmud · Roydon Andrew Fraser
}

Received: 23 January 2006/ Accepted: 9 February 2006/Published online: 26 October 2006

(C) Springer-Verlag 2006

\section{Heat Mass Transfer (2005) 41(11):999-1003}

The authors regret that the following typos occurred in the above article:

1. Equation 7: The right-hand side should be multiplied by 4 instead of the left-hand side

$$
U \frac{\partial \Theta}{\partial X}=4 \frac{1}{R^{j}} \frac{\partial}{\partial R}\left(R^{j} \frac{\partial \Theta}{\partial R}\right)
$$

2. Equation 9: The second term on the right-hand side should be multiplied by 16

$$
\begin{aligned}
\Theta= & \frac{(m+j+1)(j+1)}{m}\left[\frac{R^{2}}{2(j+1)}-\frac{R^{m+2}}{(m+j+1)(m+2)}\right] \\
& +\frac{16 X}{2(2-j)}+\Gamma
\end{aligned}
$$

3. Equation 13: The right-hand side should be multiplied by 16

$\Theta_{\mathrm{m}}=-\frac{16}{2} \frac{X}{j-2}$

4. Equation 16: $15 j^{2}$ at the denominator should be replaced by $5 j^{2}$

$\mathrm{Nu}_{\infty}=\frac{2(2-j)(3+j)(3+m+j)(3+2 m+j)}{2 m^{2}+7 j m+13 m+17+18 j+5 j^{2}}$. 